\title{
ESTUDO COMPARATIVO ENTRE OS METODOS DE STOLL E KATO QUANTITATIVO
}

\author{
Ogvalda Devay*
}

\begin{abstract}
A autora realizou a contagem de ovos por gr. de fezes em 129 amostras de fezes pelos métodos de Kato e de Stoll, incluindo 100 casos de parasitismo por Ascaris lumbricoides, 100 por Trichuris trichiura, 70 por ancilostomideos $e$ por S. mansoni.

Os resultados foram avaliados estatisticamente, evidenciando-se idênticos com as duas técnicas no que se refere a Ascaris lumbricoides, T. trichiuris $e$ S. mansoni, mostrando-se, no entanto, o método de Stoll mais adequado para a contagem de ovos de ancilostomideos.
\end{abstract}

\section{INTRODUÇĀO}

muito importante, no estudo das helmintoses intestinais, o exame coproscópico quantitativo, principalmente na investigação epidemiológica ou terapêutica (10).

O trabalho científico não pode ser devidamente apreciado se utiliza técnica não muito conhecida ou avaliada.

Em 1923, surge uma técnica de contagem de ovos de helmintos por grama de fezes, evidenciando, com precisão, ovos de Ascaris lumbricoides, Trichocephalus trichiurus, Schistosoma mansoni, tão bem quanto de Ancilostomídeos, técnica que se tornou clássica, utilizada em todos os trabalhos científicos que requeriam o procedimento de contagem de ovos por grama de fezes (9)
Em 1954, Kato e Miura introduziram um método coproscópico em ovohelmintoscopia que, apreciado em 1966 por Komyia e Kobayashi (7), e em 1968 por Martin e Beaver (8), se revelou muito sensível para detectar todos os ovos comuns de helmintos intestinais nas fezes.

Em 1969, Callot e col. (2) compararam a técnica de Kato com a de Faust e col. e concluíram ser aquela de excelentes resultados para ovos de helmintos.

Tem-se usado em inquéritos coprológicos quantitativos, portanto, clássicamente, a técnica de Stoll. Resolvemos comparar os resultados obtidos pelas técnicas de Stoll e Kato, a fim de que pudéssemos utilizar esta última em levantamento epidemiolólógico no subúrbio de Periperi, Salvador-Bahia.

\footnotetext{
- Prof.n Assistente de Parasitologia. 
TABELA I - ANÁLISE QUALITATIVA DOS MÉTODOS DE KATO E STOLL

\begin{tabular}{l|c|c|c|c|c}
\hline \multirow{2}{*}{ HELMINTO } & \multirow{2}{*}{$\begin{array}{c}\text { N. } \\
\text { DE }\end{array}$} & \multicolumn{3}{|c}{ TÉCNICA EMPREGADA } \\
\cline { 3 - 5 } & EXAMES & \multicolumn{2}{|c|}{ Kato } & Stoll \\
\cline { 3 - 5 } & & Positivos & Negativos & Positivos & Negativos \\
\hline A. lumbricoides & 100 & $97(97 \%)$ & $3(3 \%)$ & $89(89 \%)$ & $11(11 \%)$ \\
T. trichiurus & 100 & $99(99 \%)$ & $1(1 \%)$ & $87(87 \%)$ & $13(13 \%)$ \\
S. mansoni & 70 & $57(81 \%)$ & $13(19 \%)$ & $54(77 \%)$ & $16(22 \%)$ \\
Ancilostomideos & 40 & $35(87 \%)$ & $5(12 \%)$ & $15(37 \%)$ & $25(62 \%)$ \\
\hline
\end{tabular}




\section{MATERIAL E MÉTODOS}

O material utilizado procedeu, na quase totalidade, de escolares de 7 a 14 anos do subúrbio de Periperi. As fezes foram coletadas em latas de metal, distribuídas nas escolas públicas estaduais e municipais.

Da mesma amostra recebida na lata, do mesmo bolo fecal, portanto, no mesmo dia da coleta, procedeu-se à contagem dos ovos de helmintos pelas duas técnicas, a de Kato e a de Stoll. Foram realizados exames de 129 amostras. De cada amostra se preparou uma única lâmina pelo método de Kato, usando-se $50 \mathrm{mg}$ de fezes, e se retiraram $4 \mathrm{~g}$ para preparar o stoll.

Para o método de Stoll seguimos a técnica clássica (9). Para o método de Kato, também utilizamos a técnica clássica (6), introduzindo, contudo, um rolo compressor de madeira - após cobrir o material fecal com a laminula de celofane, comprimíamos, delicadamente, a lamínula contra o esfregaço com o auxílio de um compressor cilíndrico de madeira, com peso de $44,4 \mathrm{~g}$ e dimensão łle $3,5 \mathrm{~cm}$ de diâmetro, $4 \mathrm{~cm}$ de altura, com um cabo de $11 \mathrm{~cm}$.

\section{RESULTADOS}

Das 129 amostras examinadas, reunimos 100 casos positivos para Ascaris lumbricoides, 100 para Trichocephalus trichiurus, 70 para Ancilostomídeos e $\mathbf{4 0}$ para Schistosoma mansoni.

Conforme a Tabela I, em 100 amostras, para o Ascaris lumbricoides, 97 foram positivas pelo Kato ( 3 negativas) e 89 pelo Stoll (11 negativas). Em 100 amostras para o Trichocephalus trichiurus, 99 foram positivas pelo Kato (apenas 1 negativa), e 87 pelo Stoll (13 negativas). Em amostras para os Ancilostomídeos, 57 foram positivas pelo Kato (13 negativas), e 54 pelo Stoll (16 negativas), em 40 amostras para Schistosoma mansoni, 35 foram positivas pelo Kato (5 negativas) e 15 pelo Stoll (25 negativas).

Conforme a Tabela II, observou-se:

Ascaris lumbricoides: nas amostras positivas pelo Kato (97), o total de ovos encontrados foi 1.432 .880 (média de 14.328,8/ (exame) e pelo Stoll (89 amostras), 1.339.860 (média de 13.398,6/exame) Em
51 amostras o número de ovos foi maior pelo Kato, e em 34 amostras foi maior o número de ovos pelo Stoll. Em um caso houve igualdade dos resultados pelos 2 métodos.

Os resultados obtidos pelos métodos de Kato e Stoll foram analisados estatisticamente, utilizando-se teste de comparação entre médias para amostras correlatas ao nível de 0,05 de significância. O teste de significância foi igual a 0,575 para Ascaris lumbricoides.

Trichocephalus trichiurus: nas amostras positivas pelo Kato (99) o total de ovos encontrados foi 353.510 (média de $3.535,1$ /exame), e pelo Stoll (87 amostras), 318:400 (média de 3.184,0/exame). Em 53 amostras o número de ovos foi maior pelo Kato e em 32 amostras foi maior o número de ovos pelo stoll. Houve igualdade de resultados em 1 caso.

Da análise estatística, o teste de significância ao nivel de 0,05 foi igual a 0,480 para Trichocephalus trichiurus.

Ancilostomídeos: nas amostras positivas pelo Kato (57) o total de ovos encontrados foi de 27.840 (média de 397,71/exame) e pelo Stoll (54 amostras positivas), 50.700 (média de 724,28/exame). Em 12 amostras o número de ovos foi maior pelo Kato, e em 20 amostras foi maior o número de ovos pelo Stoll. Em uma amostra houve igualdade dos resultados pelos 2 métodos.

$O$ teste de significância a nível de $\mathbf{0 , 0 5}$ foi igual a 2,287.

Schistosoma mansoni: nas amostras positivas pelo Kato (35) foi encontrado um total de 7.300 ovos (média de 182,5/exame). Pelo Stoll, um total de 5.000 ovos em 15 amostras positivas (média de 125,0/ /exame). Em 3 amostras o número de ovos foi maior pelo Kato, e em 7 amostras foi maior pelo stoll. Não houve igualdade de resultados pelos 2 métodos.

O teste de significância obtido, a nível de 0,05 , foi de 1,433 .

Em avaliação quantitativa, para o $A s$ caris lumbricoides, nas amostras positivas, o total de ovos encontrados por grama de fezes variou de 20 a 69.520; para Trichocephalus trichiurus, variou de 20 a 45,220 por exame; para os Ancilostomídeos, de 20 a 6.200, e para o $S$. mansoni, de 20 a 2.060 ovos por grama, pelo método de Kato. 
TABELA II - ANÁLISE QUANTITATIVA DOS MÉTODOS DE KATO E DE STOLL

\begin{tabular}{|c|c|c|c|c|c|c|c|c|c|c|c|c|}
\hline \multirow{3}{*}{ HELMINTO } & \multirow{3}{*}{$\begin{array}{c}\text { N.o } \\
\text { de } \\
\text { Exames }\end{array}$} & \multicolumn{10}{|c|}{ TENICA EMPREGADA } & \multirow{3}{*}{$\begin{array}{c}\text { Teste } \\
\text { de } \\
\text { Signifi- } \\
\text { cância } \\
\text { a nivel } \\
\text { de } \\
\mathbf{0 , 0 5}\end{array}$} \\
\hline & & \multicolumn{5}{|c|}{ KATO } & \multicolumn{5}{|c|}{ \$TOLL } & \\
\hline & & $\begin{array}{c}\text { Total } \\
\text { de } \\
\text { ovos }\end{array}$ & $\begin{array}{c}\text { Média } \\
\text { ovos } \\
\text { p/exame }\end{array}$ & $\begin{array}{c}= \\
\text { Stoll }\end{array}$ & $\begin{array}{c}\text { Kato } \\
>\end{array}$ & $\begin{array}{c}\text { Kato } \\
<\end{array}$ & $\begin{array}{c}\text { Total } \\
\text { de } \\
\text { ovos }\end{array}$ & $\begin{array}{c}\text { Média } \\
\text { ovos } \\
\text { p/exame }\end{array}$ & $\begin{array}{c}= \\
\text { Kato }\end{array}$ & $\begin{array}{c}\text { Stoll } \\
>\end{array}$ & $\begin{array}{c}\text { Stoll } \\
<\end{array}$ & \\
\hline A. lumbricoides & 100 & 1432880 & 14328,8 & 1 & 51 & 45 & 1359860 & 13398,6 & 1 & 34 & 54 & 0,575 \\
\hline T. trichiurus & 100 & 353510 & 3535,1 & 1 & 53 & 46 & 318400 & 3184,0 & 1 & 32 & 54 & 0,480 \\
\hline Ancilostomideos & 70 & 27840 & 397,71 & 1 & 12 & 44 & 50700 & 724,28 & 1 & 20 & 33 & 2,287 \\
\hline S. mansoni & 40 & 7300 & 182,5 & 0 & 3 & 32 & 5000 & 125,0 & $\theta$ & 7 & 8 & 1,433 \\
\hline
\end{tabular}


Pelo método de Stoll, a contagem de ovos por grama de fezes para o $\boldsymbol{A}$. lumbricoides variou de 100 a 92.100; para o $T$. trichiurus, de 100 a 61.900; para Ancilostomídeos, de 100 a 8.900 , e para $S$. mansoni, de 100 a 1.200 .

\section{COMENTARIOS}

Para todos os helmintos examinados, notamos que a técnica de Kato, no aspecto qualitativo, detectou maior número de casos positivos. Para o Ascaris lubricoides, em 100 casos, como se vê na Tabela I, 97 foram positivos pelo Kato, e somente 89 pelo Stoll. Essa diferença se repetiu para o Trichocephalus trichiurus (99 casos positivos pelo Kato e somente 87 pelo stoll) . Para os Ancilostomídeos, o índice de positividade não diferiu muito. Ainda assim, - Kato detectou maior número de parasitados - em 70 casos, 57 positivos pelo Kato e 54 pelo Stoll. Para o schistosma mansoni, essa diferença foi muito acentuada; em 40 casos, o Kato detectou 35 exames positivos, e o Stoll apenas 15 .

Da análise estatística, utilizando-se teste de comparação para amostras correlatas ao nivel de 0,05 de significância, chegou-se ao seguinte resultado: para $A$. lumbricoides, o valor do teste, 0,575. Para o $\boldsymbol{T}$. trichiurus, $\mathrm{t}=0,480$; para $S$. mansoni, $\mathrm{t}=1.433$. Todos esses valores, não significativos, conduzem-nos à interpretação de que os méto. dos em estudo se equivalem. Para Ancilostomideos, contudo, $\mathrm{t}=2,287$ demonstra ser o Stoll mais indicado para a contagem dos ovos desses helmintos.

\section{CONCLUSOES}

1 - Os métodos de Kato quantitativo e Stoll equivalem-se na determinaçāo do número de ovos de Ascaris lumbricoides, Trichocephalus trichiurus e Schistosoma mansoni, eliminados por grama de fezes.

2 - O método de Stoll é mais sensível que o de Kato na determinação do número de ovos de Ancilostomideos eliminados por grama de fezes.

3 - Para análise qualitativa, o método de Kato é mais sensível que o de Stoll no diagnóstico parasitológico de ascaridiose, tricocefalose, ancilostomose e esquistossomose.

\section{AGRADECIMENTOS}

O autor agradece a colaboração que the deram o Serviço de computação eletrônica e o pessoal docente da Estatística do Instituto de Matemática da Universidade Fe. deral da Bahia, na computação de dados e análise estatística dos resultados. Agradece, ainda, ao Prof. Air Colombo Barreto.

\section{$S U M M A R Y$}

The author examined 129 specimens of faeces using the techniques of Kato and Stoll. 100 cases of Ascaris lumbricoides infection, 100 of Trichocephalus trichiurus, 70 of hookworm infection and 49 of Schistosoma mansoni were de. tected. The number of eggs eliminated per gram of faeces was determined using the two methods. The results were statistically analysed using the comparative tests for the two methods at a significance level of 0,05 . The results showed that, for counting eggs, the two techniques presented equal results for A lumbricoides, $T$. trichiurus and Schistosoma mansnni, but the Stoll technique was more sensitive for hookworms 


\section{REFERENCIAS BIBLIOGRÁFICAS}

1. BELI, D. R. - A new method for counting Schistosoma mansoni eggs in feces. Bull. World Health organ. 29: 525-530, 1963.

2. CALLOT, J et alli - Kato's excellente coprologic technique. Bull. Soc. Pathol. Exot. 62: 747-50, 1969.

3. GOEDBLOED, E. et alli - Comparison of two methods for the detection of Schistosoma mansoni eggs in stools. Trop. Geog. Med., 22: 472-5, 1970.

4. KATZ, N. \& CHAIA, G. - Cropological diagnosis of schistosomiasis. I Evaluation of quantitative techniques. Rev. Inst. Med. trop. S. Paulo 10: 295-298, 1968.

5. KATZ, N. et al. - Evaluation of Kato' quantitative method through the recovery os Schistosoma mansoni eggs added to human feces. J. Parasitol. 56: 1032-1033, 1969 .
6. KATO, K - A correct application of the thick smear technic with cellophane paper cover. A F'amphlet, 1-9 (Jap. text), 1960.

7. KOMIYA, Y. \& KOBAYASIH, A. Evaluation of Kato's thick smear technic with a cellophane cover for helminth eggs in feces. Jap. J. Parasitol. 19: 59-64, 1966.

8. MARTIN, C. \& BEAVER, P. - Evalua tion of Kato thick smear technique for quantitative diagnosis of helminth infections. $A m:$ J. Trop. Med. Hyg 17: 382-391, 1968.

9. STOLL, N. R., - Investigation on the control of hookworm disease. XV. An effective method of counting bookworm eggs in feces. Am. Journ Hyg. 3: 59-70, 1923.

10. WHO, Série Informes Técnicos, 1966. 